\title{
«Ich bin kein Präsident für schwarze Schafe»
}

\section{Daniel Lüthi}

Freier Journalist und Fotograf, Medientrainer, Bern

Der Ort passt. Ein nobles Hotel mit einem grossen Seminarraum im ersten Stock, ganz in der Nähe des Bahnhofs. Dies ist jetzt die Welt von Josef Brandenberg, nicht mehr die Praxis und das Spital: unterwegs sein zu Besprechungen, Verhandlungen, Seminaren, Kongressen. Standesvertreter sein. Die grosse Politik interessiere ihn nicht, sagt er mit Nachdruck, «aber standespolitisch will ich mitreden».

\section{Kleider machen Leute}

Wer ihm im noblen Hotel begegnet, denkt auch: Das passt. Josef Brandenberg ist ein gepflegter, ein distinguierter Herr. Die Krawatte sitzt und passt selbstverständlich zur Pochette, das gestickte Monogramm auf den Manschetten harmoniert mit den entsprechenden Knöpfen. «Doch, doch, ich achte schon auf Ästhetik»,

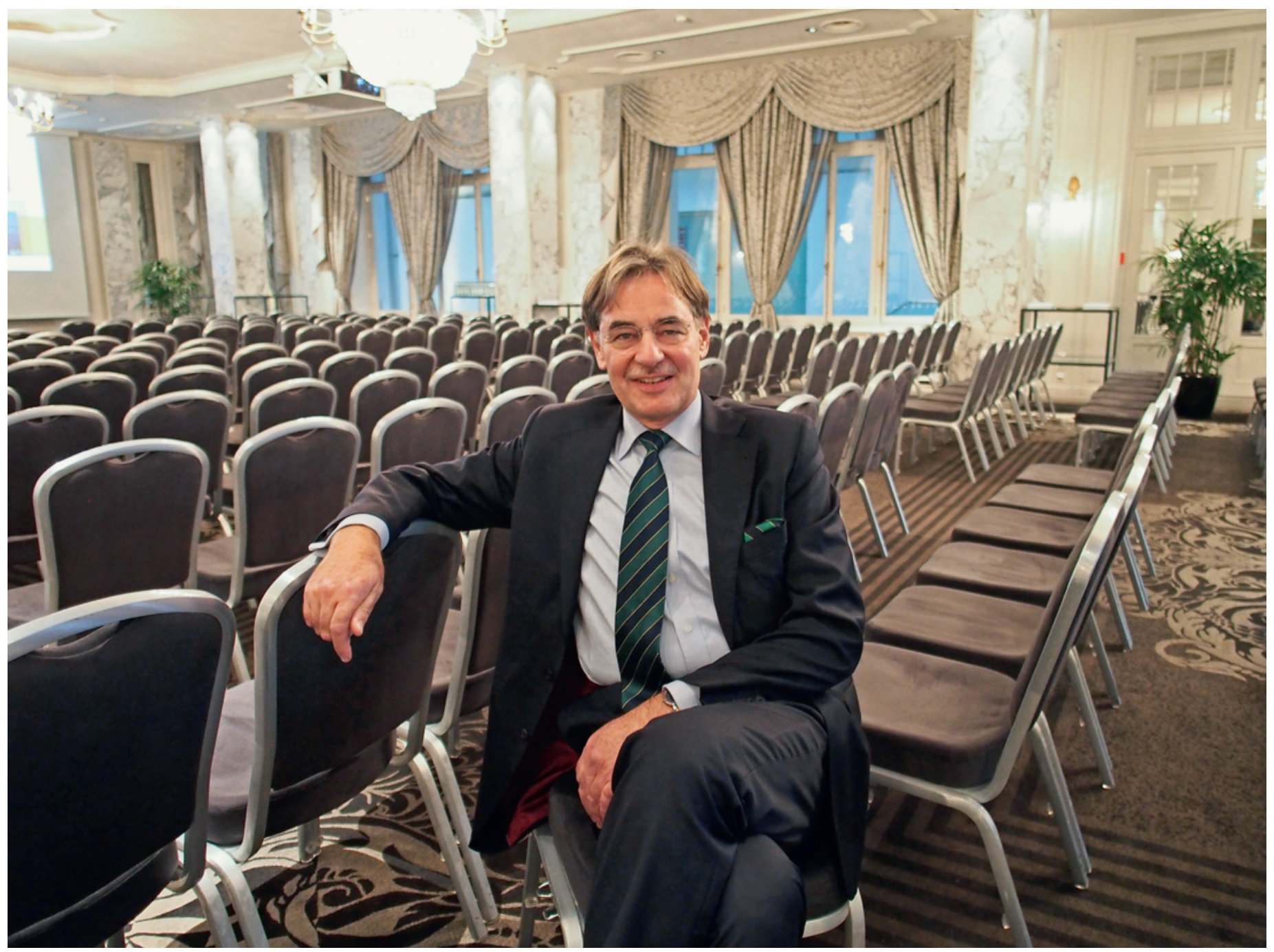


sagt er. "Und ich kleide mich gerne situationsgerecht.» Auch als er noch praktizierender Chirurg gewesen sei, habe er sich immer situationsgerecht gekleidet: "Weisse Hose, weisser Kittel, weisses Hemd - das war für mich in der Praxis und auf der Visite immer klar», sagt Brandenberg, «ich war nie ein Freund der Anbiederung.» Weiss sei für ihn "ein Ausdruck klinischer Reinheit», und er schätze es, wenn man eine Autorität an ihrem Äusseren gleich erkenne: «Früher wusste man noch, wer der Briefträger, der Polizist, der Pfarrer oder eben der Doktor ist, heutzutage kaum mehr.» Mit einer Verherrlichung der Zeit, als ein Arzt noch als «Halbgott in Weiss» wahrgenommen werden wollte, habe dies nichts zu tun. "Ich will meine Erscheinung nicht als Machtinstrument einsetzen. Aber ich weiss, dass meine Patientinnen und Patienten es schätzten, dass der Herr Doktor als Doktor noch erkennbar war. Sie haben sich mir auch im Bewusstsein anvertraut, es mit einer fachlichen Autorität zu tun zu haben.» Als grosser Fan von klassischer Musik schätze er es an einem Konzert übrigens auch, wenn nicht nur die Musiker auf der Bühne, sondern auch die Leute im Saal festlich gekleidet seien - «damit in der Wahrnehmung kein Bruch entsteht».

\section{Zunehmende Spezialisierung}

Als Orthopäde erlebte Brandenberg in den vergangenen Jahren einen starken Wandel seines Fachs. «Er bestand vor allem in einer zunehmenden Spezialisierung, die auch unsere Gruppenpraxis prägte», erzählt Brandenberg. "Einer war auf Fusschirurgie spezialisiert, einer auf die oberen Extremitäten, und ich auf die unteren Extremitäten.» Konkret: Josef Brandenberg operierte vor allem Knie- und Hüftgelenke. «Gerade in einem handwerklichen Fach ist eine Spezialisierung richtig», sagt er, "das bedeutet nämlich auch: Von diesem oder jenem verabschiede ich mich. Zu Beginn hatte ich beispielsweise noch die Verpflichtung, Diskushernien zu operieren. Aber die Wirbelsäulenchirurgie nahm ihren Lauf mit neuen Techniken und neuen Implantaten, da konnte ich nicht Schritt halten.»

Umso lieber erzählt er von den technischen Fortschritten in seiner Nische: «Der Einsatz von Navigationsgeräten in der Endoprothetik ist faszinierend: Sensoren am Beckenrand und auf dem Oberschenkel rechnen das Hüftdrehzentrum aus. Die Daten kommen als Bild auf den Schirm - das ist meine Ausgangssituation für die Operation.»

War - denn seit vier Jahren operiert Brandenberg nicht mehr.

\section{Josef E. Brandenberg}

Dr. med. Josef E. Brandenberg wurde 1950 in Baar geboren. Sein Medizinstudium an der Universität Zürich schloss er 1976 mit dem Staatsexamen ab. Die Ausbildung zum Facharzt für Orthopädische Chirurgie und Traumatologie des Bewegungsapparates führte ihn nach Zug, Locarno, Lugano und in die Klinik Balgrist in Zürich. 1987 bis 2006 führte

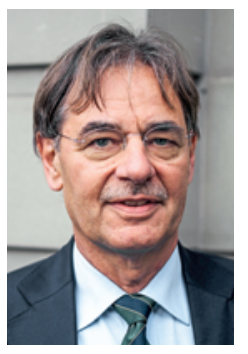
er eine Einzelpraxis in Luzern, 2006 gehörte er zu den Gründungsmitgliedern der Orthopädischen Klinik Luzern AG. Bis 2012 operierte er an der Hirslanden Klinik St. Anna in Luzern. 2014 wurde er zum President elect der fmCh bestimmt, seit Beginn dieses Jahres ist er als Präsident dieses Fachverbandes im Amt. Josef Brandenberg ist verheiratet und Vater von zwei erwachsenen Söhnen. Er lebt in Luzern.

\section{Abschied vom Ops}

«Es war ein bewusster Entscheid - und es war eine Erleichterung.» Der Abschied vom Operieren hatte vor allem mit den Komplikationen oder möglichen Komplikationen zu tun, die damit verbunden sind. «Es gibt nur einen Chirurgen, der nicht mit Komplikationen zu kämpfen hat», sagt Brandenberg, "das ist der pensionierte Chirurg. Selber entwickelte ich eine schleichende Aversion gegen klagende Patienten. Und bevor ich säuerlich zu reagieren begann, hörte ich lieber auf.» Wobei der Begriff «klagen» durchaus in seinen beiden Bedeutungen zu verstehen ist: «sich beschweren» und "gerichtlich vorgehen». "Selber hatte ich nicht überdurchschnittlich viel Pech», konstatiert er. "Aber einiges habe ich schon gesehen. Und einiges auch beheben müssen. Einen Schadenfall hatte ich, bei dem

"Gerade in einem handwerklichen Fach ist eine Spezialisierung richtig.»

meine Versicherung zahlen musste.» Eine gewisse Abgeklärtheit ist bei Josef Brandenberg spürbar, wenn er sagt: «Über Behandlungsfehler spricht niemand gern. Aber sie gehören dazu.» Im Februar referiert er an einem Kongress in Berlin über Gefässverletzungen bei Hüft- und Knieoperationen. «Das sind bekannte Komplikationen. Mit denen müssen wir leben.» Nervenverletzungen gehörten dazu, sagt Brandenberg, und Frakturen, die nicht zusammenwachsen wollen. «Das kann einen Chirurgen bedrücken. Ich habe vor einem Operationstag nie gut geschlafen.»

\section{Berufspolitischer Kämpfer}

Schon als Assistenzarzt im Tessin hat sich Josef Brandenberg «auch an der Peripherie» engagiert, wie er es 
nennt. Beispielsweise setzte er sich für NachtdienstEntschädigungen ein und eine grosszügige Interpretation des Zusatzes «wenn der Betrieb sie erlaubt». Im Alter von 28 Jahren sprach er in dieser Sache beim Spitaldirektor vor. "Ja», kommentiert Brandenberg, «berufspolitisch war ich immer ein Kämpfer.»

2014 bereits war klar, dass er dereinst Nachfolger des abtretenden charismatischen fmCh-Präsidenten Urban Laffer werden würde. Im Dezember fand in Biel die Amtsübergabe statt, seit Jahresbeginn steht Brandenberg offiziell an der Spitze dieses Verbandes. Indirekt macht er klar, dass die Fussstapfen, die der abgetretene Präsident nach zwölf Jahren hinterlässt, gross sind. «Ein neuer Besen wischt besser», sagt er, «aber der alte weiss, wo der Dreck ist.» Dezidiert will er sich für ein besseres Image der Chirurgen einsetzen. «Wir waren in den vergangenen Jahren nur noch Prügelknaben", stellt er fest, "das muss aufhören. Unnötige Operationen verschwinden fast von selber, im Rahmen eines wissenschaftlichen Prozesses.» So seien früher Kinderhüften zu Tausenden operativ korrigiert worden, "später merkte man, dass das nichts bringt. So verschwand diese Operation vom Erdboden".

Weitere Kernthemen seien aus seiner Sicht die Sicherung des Nachwuchses und die Sicherung der Qualität.

\section{... und das liebe Geld}

In der Kritik stehen die Chirurgen immer wieder wegen ihres Einkommens. «Ich distanziere mich von Exzessivem, wehre mich vehement gegen Abzocker», konstatiert Brandenberg. «Ich bin kein Präsident für schwarze Schafe.» Aber die Entlöhnung sei nur mit einem Bezug zur Arbeits- und Lebenszeit zu beurteilen. Zu Spitzenzeiten habe er gut verdient, ja, sagt Brandenberg, aber auch viel gearbeitet. «Dazu stellt sich natürlich die
Frage, wie lange die Lebensphase dauert, in der ich ein solches Einkommen überhaupt realisieren kann. Eine Studie hat gezeigt, dass ich - bezüglich Einkommen übers Ganze gerechnet besser Mittelschullehrer geworden wäre.» Und der Einkommensgraben zwischen Spezialisten und Grundversorgern? Eine Umverteilung komme für ihn nicht in Frage, «das wäre willkürlich.

\section{«Über Behandlungsfehler spricht niemand gern."}

Kostenneutralität geht ebenfalls nicht. Ich kenne einige Hausärzte, die gut verdienen», sagt der Orthopäde, «und die nicht denken, ein Missgunst- und Neidverhalten propagieren zu müssen. Auf der anderen Seite sehe ich schon, dass die Grundversorger seit der Einführung des neuen KVG keine Möglichkeit mehr haben, Zusatzhonorare zu verlangen.» Das sei bei ihm anders gewesen. «Im Spital haben sich die Zusatzversicherungen für mich ausbezahlt.»

Und wo hat der neue fmCh-Präsident sein Geld investiert? In die Ausbildung seiner beiden Söhne, sagt er, in den Bau eines eigenen Hauses, in Konzerte und Theateraufführungen. Ein Segelschiff habe er nie gehabt, «und Autos sagen mir nicht viel. Ich fahre immer noch den VW Golf Cabriolet, den ich zu meinem Vierzigsten erhalten habe.»

Udo Jürgens singt: «Mit 66 Jahren, da fängt das Leben an.» Der 66-jährige Josef Brandenberg sagt: «Eine neue Epoche hat begonnen. Früher war ich bis zur letzten Minute verplant. Das ist heute nicht mehr so.» Für seine Söhne hätte er sich gerne mehr Zeit genommen, damals. Für sein Grosskind, ein eineinhalbjähriges Mädchen, nehme er sie sich jetzt.

\section{Bildnachweis}

Fotos: Daniel Lüthi 